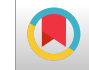

\title{
Association Between Geographic Tongue and Personal Temperament Based on the Knowledge of Persian Medicine
}

\author{
Sara Pourshahidi ${ }^{1}$, Arash Mansourian ${ }^{1}$, Maryam Habibzadeh ${ }^{2}$, Mohammad Mahdi Parvizi $\mathbb{i}^{3,4,}{ }^{*}$ and \\ Majid Nimrouzi ${ }^{5,4}$ \\ ${ }^{1}$ Oral Medicine Department, Faculty of Dentistry, Tehran University of Medical Sciences, Tehran, Iran \\ ${ }^{2}$ Faculty of Dentistry, Tehran University of Medical Sciences, Tehran, Iran \\ ${ }^{3}$ Molecular Dermatology Research Center, Shiraz University of Medical Sciences, Shiraz, Iran \\ ${ }^{4}$ Essence of Parsiyan Wisdom Institute, Phytopharmaceutical and Traditional Medicine Incubator, Shiraz University of Medical Sciences, Shiraz, Iran \\ ${ }^{5}$ Department of Traditional Persian Medicine, School of Medicine, Shiraz University of Medical Sciences, Shiraz, Iran \\ "Corresponding author: Molecular Dermatology Research Center, Shiraz University of Medical Sciences, Zand Ave, P.O. Box: 7134844119, Shiraz, Iran. Tel: +98-7132125592; Fax: \\ +98-7132319049, Email: mmparvizi@gmail.com
}

Received 2020 March 09; Revised 2020 April 26; Accepted 2020 June 03.

\begin{abstract}
Background: Geographic tongue (GT) is one of the benign inflammatory oral diseases with unknown etiology.

Objectives: The present study aimed to find the correlation between temperament (mizaj) and GT lesions in the context of Persian medicine and conventional approach.

Methods: In this cross-sectional study, 401 eligible participants were selected, 201 as the case group and 200 as the control group. After obtaining informed consent, the participants were asked to fill out a questionnaire. Then, the collected data were analyzed using SPSS, version 21.

Results: Patients with GT were moderate regarding hot-cold temperament, and they had dry temperament. There was a statistically significant relationship between dry temperament and GT lesions (P value $=0.037$ ). In the control group, people without GT lesion possessed average temperament either regarding hot-cold or wet-dry temperament. Moreover, there was a statistically significant relationship between hot-dry temperament and GT lesions (P value $=0.002$ ).

Conclusions: The results of our study showed a higher frequency of GT in dry temperament. The dominant pattern for people with GT in compound and simple temperament was hot-dry and dry, respectively. The second more frequent temperament was hot. Following temperament change, treatment of GT lesion or alleviation of its disturbing symptoms is an issue, which might be determined by conducting basic research and well-designed clinical trials in the future.
\end{abstract}

Keywords: Tongue, Glossitis, Traditional Medicine, Temperament

\section{Background}

The tongue has traditionally been known as a marker of primary oral hygiene and general health (1). As a pivotal organ in oral function, it may be afflicted with various anomalies and disorders, leading to malfunction and tongue symptoms, such as burning and pain (2). Geographic tongue (GT), also known as benign migratory glossitis, is one of the tongue disorders $(3,4)$, whose development may affect the patient's beauty and anguish even some affected people due to its malignant change $(5,6)$.

Precise clinical examination of the lesion and definite diagnosis may calm the patients and reduce their suffering (7). Benign inflammatory patches are relatively common in GT (8-11). These lesions are usually multi-pitted, semilu- nar, or irregular, and without filiform papillae. Their margins are prominent in white or yellow color $(5,12)$. Lesions are inclined to change their location, pattern, and size. All areas in the back or sides of the tongue's epithelium may be involved. It shows that remission exacerbation periods and remission last for days, months, or years without any scar trace. Relapse of lesions can occur in new places that demonstrate a migratory feature to GT $(5,13)$. Lesions are usually asymptomatic, of which patients are often unaware $(7,14)$. However, they are sometimes sensitive to sour, spicy, or hot food $(15,16)$. The prevalence of GT was reported differently in various populations, such as $1.5 \%$ in the Turkish population (17), $1.8 \%$ in the United States population (18), 17.2\% in Libyan adult population (19), and $35.11 \%$ in Hungarian children population (20). Moreover, 
the prevalence of GT in Iran was 7.6\% in student children in Rasht, Northern Iran (21), 7.86\% in student children in Kermanshah, Western Iran (22), and 7.8\% in adult dental patients in Zahedan, Southeastern Iran (4). The vast range of reports is probably due to several factors, including ethnic groups, sex, and age of study samples, different diagnosis criteria, as well as the method of examination and sampling $(19,23)$.

In this regard, some evidence showed that GT was more common in children, and the incidence rate decreases with increasing age; According to literature, the mean age of people with GT was about 23 years old in the Iranian population (24). A few evidence showed that GT was two times more common in women (25); however, other studies demonstrated the same prevalence in both sexes $(17,18)$.

Despite increasing advances in conventional medicine, there are many unresolved problems, especially in the control and treatment of chronic diseases. There is an increasing inclination toward the alternative approach and integrative medicine throughout the world (26). Persian medicine (PM), one of the most significant branches of complementary medicine, has been regarded as a valuable medical resource throughout the world, and many Iranian people provided their medical demand via PM routes, i.e., referring to herbalist, local PM therapist, or using folk medicine recommendations $(27,28)$. Mezaj (temperament) is a crucial point in health and is regarded as a serious diagnostic tool for PM physicians (29-31). Based on the PM approach, everybody should observe health measures, including dietary, physical activity, sleep, and mental health, to maintain their body organs balanced to set all normal functions of the body in an optimum state. In other words, everybody is based on constitutional temperament, with observing dietary measures, maintain their primary constitutional temperament in balance, and prevent any sudden intensive changes in quadruple qualities leading to dystemperament. In this regard, the organs' function would be set in the optimum state physically and mentally $(32,33)$.

To consider the temperament for all disease states is a key point in the context of the PM research in order to acquire more effective therapy with fewer side effects through tailoring to the temperament-oriented medical intervention. To the best of our knowledge, there was no evidence about the relationship between GT and temperament in the literature review of conventional medicine.

\section{Objectives}

Thus, the present study aimed to evaluate the temperament of patients with GT who went to the Faculty of Dentistry affiliated to Tehran University of Medical Sciences and compare it with the temperament of individuals without GT.

\section{Methods}

\subsection{Design of the Study, Participants, and Sample Size}

In this descriptive-analytical crossectional study, all the participants were selected from those who were taken to the Faculty of Dentistry, Tehran University of Medical Sciences, from March 2016 to November 2016 for dental care. They were examined by an oral medicine specialist via the dentistry mirror and sterile gas. The minimum sample size of 401 participants ( 200 participants in each group) was calculated for this study with considering $\mathrm{P}=5 \%$, a $95 \%$ confidence interval, and a $5 \%$ alpha error by conducted this formula:

$n=\frac{P(1-P) Z^{2}}{d^{2}}$

We selected 401 eligible participants, including 201 patients with diagnosis of GT based on clinical examination as the case group and 200 patients without GT as the control. After obtaining informed written consent, we asked them to fill out the questionnaire. None of the patients in both groups had a history of discomfort, burning sensation, pain, etc.

\subsection{Inclusion and Exclusion Criteria}

The inclusion criteria were the 20 - 40 years old individuals who referred to the Faculty of Dentistry for routine dental visits and the dentist-approved GT in them. The exclusion criteria were individuals with a positive history of any apparent diseases including diabetes, psoriasis, allergy, Reiter disease, pregnancy, and other known chronic or acute diseases, as well as the patients who had the lack of willingness to continue participating in the project and lack of cooperation to fill out the questionnaire.

\subsection{Ethics Statements}

The protocol of the study was approved by the Ethics Committee of Tehran University of Medical Sciences (ethics code: 8911272080 ). Before asking the participants to sign the informed consent, we informed them about the study process. 


\subsection{Questionnaire}

The participants filled out the Mojahedi et al.'s (34) questionnaire (with Cronbach's alpha $=0.71$ ), which was valid and reliable for the age group of 20 - 40 years. Data gathered from the patients were kept confidential, and the researcher responded to the questions of the patients.

It comprised ten questions for determination of temperament (eight for evaluation of hotness-coldness and the remaining two for evaluation of wetness-dryness), which was designed according to the Likert scale (1 - 3 score). The total score of the first eight questions showed hot, cold, and the average temperament if it was $>19,<14$, and $15-18$, respectively. The total score of the last two questions showed dryness, wetness, and average in wetnessdryness if it was $>4,<4,4$, respectively.

\subsection{Statistical Analysis}

Collected data were analyzed using SPSS Version 21. Chisquare and independent-sample $t$-test were used to assess the relationship between the variables and groups. The $P$ value of less than 0.05 was considered as statistical significant.

\section{Results}

About 167 subjects (83.5\%) in the case group and 158 (78.6\%) in the control group were female. There was no statistically significant difference between the two groups regarding the sex ratio ( $P$ value $=0.2$ ). The mean age of the case and control groups was $31.4 \pm 7.2$ and $29.5 \pm 6.2$, respectively $(P$ value $=0.005)$.

Concerning hotness, both groups were average more frequently. Regarding dryness, the case group showed dry temperament more commonly, while in the control group, the average temperament dominated. Nevertheless, there was no statistically significant difference between the case and control groups in hotness ( $\mathrm{P}$ value < 0.05). The frequency of dryness in the case group was statistically significant $($ Pvalue $=0.037)$. Table 1 shows the relationship between compound temperament and GT. Comparing compound temperament in the case and control groups showed a statistically significant relationship between hot-dry temperament and GT $(\mathrm{P}=0.002)$.

\section{Discussion}

GT patients with positive history of disease exacerbation reveal a possible temperament on remission/exacerbation of GT lesions after eating foods with special temperament. A study on 25 people, regarding the occurrence and aggravation of GT lesions, reported some foods, such as eggplants (65\%), tomato (43\%), nuts (23.3\%), pickles (14\%), spicy foods (12\%), and tuna (11\%). Nevertheless, the time between food consumption and occurrence and aggravation of lesions was uncertain for most patients; some were unaware of their problem before medical visit and disease confirmation (24).

In our study, although all patients were between 20 and 40 years old, there was a statistically significant difference between the mean age of the GT group (31 y/o) and control group $(29 \mathrm{y} / 0)$ (Pvalue $<0.05)$, which is in line with some recent research studies $(35,36)$. However, Jainkittivong and Langlais (13) and Kullaa-Mikkonen et al. (37) did not find any significant difference between the age of GT and the control group due to different research methods and population characteristics of the studies.

In this study, female patients (78\%) were more than male patients (21.4\%), which is consistent with previous studies (7, 9, 16, 37). Maleki et al. and Taheri and Maleki (38, 39) showed that the prevalence of GT was higher in girls, which is in line with the female dominancy in our study. According to some studies, the occurrence and aggravation of GT lesions and higher frequency of GT in females may be due to the contribution of female sex hormones $(36,40,41)$. Aggravation of disease has been reported in some studies with the menstrual cycle (41). Although few studies found no sex difference $(18,41)$, some reported a higher frequency of GT in males $(20,37)$. There was a similarity between case and control groups in female dominancy in our study. However, there might be other reasons such as more concern about their disease and more cooperation in filling out the questionnaire by women compared to men.

In terms of the PM approach, age is a variable that affects temperament, as a result, we selected the young age group to control for this effect on temperament (4144). Unlike the middle-aged and elderly, young people are hot-wet (45). Patients in the GT group were two years older than those in the control group, which was in line with increasing dryness in the older group. On the other hand, the place of residence is another important factor that had an effect on the temperament and can change it. In our study, the participants in both groups were homogeneous in this factor because all of them were lived in Tehran. According to PM sources, systemic diseases can affect temperament. For instance, patients with allergy, Reiter's disease, and psoriasis are more likely to be affected by hot-temperament of the liver, where black bile produc- 


\begin{tabular}{|c|c|c|c|c|c|}
\hline \multirow{2}{*}{ Groups } & \multicolumn{5}{|c|}{ Temperament } \\
\hline & Hot-Dry & Hot-Wet & Cold-Dry & Cold-Wet & Sum/Total \\
\hline Case & $28(13.9)$ & $10(5)$ & $20(10)$ & $8(4)$ & $58 / 201$ \\
\hline Control & $9(4.5)$ & $11(5.5)$ & $18(9)$ & $8(4)$ & $46 / 200$ \\
\hline Pvalue & 0.002 & 0.827 & 0.86 & 1.00 & \\
\hline
\end{tabular}

${ }^{\mathrm{a}}$ Values are expressed as No. (\%).

tion is increased $(46,47)$. Also, diabetes mellitus, chronic stresses, and chronic use of tranquilizers are associated with increased black bile production and the dominancy of cold/dry temperament in the afflicted patients, and the occurrence of constipation. Anemia, history of some foods, and drug consumption correlate with the dominancy of hot/dry temperament in some people $(48,49)$. In this study, we selected healthy people without a history of drug consumption to eliminate the effect of the aforementioned factors (33). Table 2 shows that most of the participants in the GT group had average temperament based on the hot/wet quality, as well as dry temperament based on the wet/dry quality. Also, dry dystemperament played a more crucial role in the occurrence of GT in people with simple dystemperament. Most of the people in the control group were on average temperament in both hot/cold and wet/dry qualities. These results further reinforce the claim of a relationship between GT occurrence and dryness. However, compound dystemperament, hot/dry temperament was more frequent in the GT group. According to PM, hot temperament may aggravate the dryness of the body and durability of hot dystemperament and cause dry dystemperament in the body (1). A few studies have focused on the relationship between temperament and medical conditions. In this regard, Parvizi et al. (50) demonstrate the positive association of neuroticism personality type with hot temperament.

PM sources also confirm the correlation of dry dystemperament and GT. There is a similar tongue disease in PM treaties and books that are related to dry dystemperament of the tongue and other body organs and presents with similar signs and symptoms including shaqaq (furrow in tongue) with dryness and yellowish color of the tongue and qelaa (ulceration) with burning sensation in the tongue. Dry dystemperament in the body, especially in the head and neck area, is the common pathology for all of the mentioned diseases. Furthermore, relatively similar treatment plans have been recommended for most patients with these diseases.

\subsection{Limitations}

To the best of our knowledge, this is the first study on the relationship between GT and temperament. Therefore, this could be a limitation for itself because all materials must be provided for the first time; however, the valid questionnaire for the temperament determination was a great support for the first step.

\subsection{Conclusions}

The present study demonstrated the relationship between hot temperament and dry temperament with GT. It is suggested that designing clinical studies be conducted in the future for better evaluation and investigation of dietary patterns and drug consumption history of GT patients as plausible causes of disease exacerbation. Given the emphasis of Iranian sages on viewing dystemperament as the cause of many diseases, studying the relationship between chronic diseases and temperament imbalance can help solve some resolved medical problems. To do this, the first step could be to design valid and reliable questionnaires that evaluate the temperament of a single body organ and the whole body.

\section{Footnotes}

Authors' Contribution: Study concept and design: Sara Pourshahidi, Arash Mansourian; Mohammad Mahdi Parvizi, and Majid Nimrouzi. Acquisition of data: Sara Pourshahidi, Arash Mansourian, and Maryam Habibzadeh. Statistical analyses: Mohammad Mahdi Parvizi, Sara Pourshahidi, and Arash Mansourian. Drafting of the manuscript: Mohammad Mahdi Parvizi, Maryam Habibzadeh, and Majid Nimrouzi. Critical revision of the manuscript for important intellectual content: Sara Pourshahidi, Mohammad Mahdi Parvizi, and Arash Mansourian. Study supervision: Sara Pourshahidi, Mohammad Mahdi Parvizi, and Arash Mansourian.

Conflict of Interests: No conflicts of interests declared. 


\begin{tabular}{|c|c|c|c|c|c|c|c|c|}
\hline \multirow{3}{*}{ Groups } & \multicolumn{8}{|c|}{ Temperament } \\
\hline & \multicolumn{4}{|c|}{ Wetness } & \multicolumn{4}{|c|}{ Hotness } \\
\hline & Dry & Moderate & Wet & Sum & Cold & Moderate & Hot & Sum \\
\hline Case & $92(45.8)$ & $69(34.3)$ & $40(19.9)$ & $201(100)$ & $44(21.9)$ & $99(49.3)$ & $58(28.9)$ & $201(100)$ \\
\hline Pvalue & 0.037 & 0.078 & 0.393 & & 0.235 & 0.049 & 0.134 & \\
\hline
\end{tabular}

${ }^{\mathrm{a}}$ Values are expressed as No. (\%).

Ethical Approval: The study was approved by the Ethics Committee of Tehran University of Medical Sciences (ethics code: 8911272080 ).

Funding/Support: This study was supported by the Research Chancellor of Tehran University of Medical Sciences, Tehran, Iran (grant no.: 6206).

Informed Consent: The enrolled participants were informed about the study, and signed informed consent was obtained from them.

\section{References}

1. Jarjani S. Zakhire-i-Kharazmshahi. Tehran: Iran's Culture Foundation Publication; 2000

2. Islam NM, Bhattacharyya I, Cohen DM. Common oral manifestations of systemic disease. Otolaryngol Clin North Am. 2011;44(1):161-82. vi. doi: 10.1016/j.otc.2010.09.006. [PubMed: 21093628].

3. Ghalyani P, Hajisadeghi S, Mokhtari H. [Prevalence and clinical symptoms of geographic tongue in pregnant women]. Dent Res J (Isfahan). 2012:251-9. Persian.

4. Honarmand M, Mollashahi LF, Shirzaiy M, Sehhatpour M. Geographic tongue and associated risk factors among Iranian dental patients. Iran J Public Health. 2013;42(2):215.

5. Assimakopoulos D, Patrikakos G, Fotika C, Elisaf M. Benign migratory glossitis or geographic tongue: an enigmatic oral lesion. Am J Med. 2002;113(9):751-5. doi: 10.1016/s0002-9343(02)01379-7.

6. Abdolsamadi H, Hamian M. An investigation on therapeutic effect of zinc sulfate in patients with geographic tongue. J Dent Med. 2005;18(4):63-8.

7. Burket LWGM, Glick M. Burket's oral medicine: diagnosis $\mathcal{E}$ treatment. Washington, DC: PMPH-USA; 2003.

8. Greenburg MGM. Burket's Oral Medicine. 9th ed. BC Decker: Hmilton; 2003. p. 115-6.

9. Laskaris G, Shklar G. Color atlas of oral diseases. Stuttgart- New York: Litas Medical; 2003. p. 120-2.

10. Scully C. Oral and maxillofacial medicine. Edinburg: Elsevier; 2005.

11. Femiano F. Geographic tongue (migrant glossitis) and psoriasis. Minerva Stomatol. 2001;50(6):213-7.

12. Goswami M, Verma A, Verma M. Benign migratory glossitis with fissured tongue. J Indian Soc Pedod Prev Dent. 2012;30(2):173-5. doi: 10.4103/0970-4388.100008. [PubMed: 22918106].

13. Jainkittivong A, Langlais RP. Geographic tongue: clinical characteristics of 188 cases. J Contemp Dent Pract. 2005;6(1):123-35. [PubMed: 15719084].

14. Ugar-Cankal D, Denizci S, Hocaoglu T. Prevalence of tongue lesions among Turkish schoolchildren. Saudi Med J. 2005;26(12):1962-7.
15. Scully C. The basis of diagnosis and treatment. Oral and maxillofacial medicine. Philadelphia: Elsevier Health Sciences; 2013. p. 10-20. doi: 10.1016/b978-0-7020-4948-4.00002-7.

16. Regezi J, Sciubba J, Jordan R. Oral pathology: Clinical pathologic correlations. New Delhi: Elsevier Health Sciences; 2016.

17. Miloglu O, Goregen M, Akgul HM, Acemoglu H. The prevalence and risk factors associated with benign migratory glossitis lesions in 7619 Turkish dental outpatients. Oral Surg Oral Med Oral Pathol Oral Radiol Endod. 2009;107(2):29-33. doi: 10.1016/j.tripleo.2008.10.015. [PubMed: 19138635].

18. Shulman JD, Carpenter WM. Prevalence and risk factors associated with geographic tongue among US adults. Oral Dis. 2006;12(4):381-6. doi: 10.1111/j.1601-0825.2005.01208.x. [PubMed: 16792723].

19. Byahatti SM, Ingafou MSH. The prevalence of tongue lesions in Libyan adult patients.J Clin Exp Dent. 2010:163-8. doi:10.4317/jced.2.e163.

20. Voros-Balog T, Vincze N, Banoczy J. Prevalence of tongue lesions in Hungarian children. Oral Dis. 2003;9(2):84-7. doi: 10.1034/j.16010825.2003.00783.x. [PubMed: 12657034].

21. Rabiei M, Mohtasham AZ, Amigh S, Ghotbirad SF, Ahsani TSD. [Prevalence of geographic tongue, fissure tongue and partial ankyloglossia among students of three stages of school in Rasht in 2003]. Majallah Dandanpizishki. 2006;18(1):30-6. Persian.

22. Rezaei F, Safarzadeh M, Mozafari H, Tavakoli P. Prevalence of geographic tongue and related predisposing factors in 7-18 year-old students in Kermanshah, Iran 2014. Glob J Health Sci. 2015;7(5):915. doi: 10.5539/gjhs.v7n5p91. [PubMed: 26156909]. [PubMed Central: PMC4803890].

23. Yarom N, Cantony U, Gorsky M. Prevalence of fissured tongue, geographic tongue and median rhomboid glossitis among Israeli adults of different ethnic origins. Dermatology. 2004;209(2):88-94. doi: 10.1159/000079590. [PubMed: 15316160].

24. Hashemipour M, Zareie M, Farrokh-gisou E, Dastbous A. Investigatin of the relationship between inflammatory disease of thetongue and the signs of allergy. Daneshvar. 2008;15(74):77-84.

25. Ching V, Grushka M, Darling M, Su N. Increased prevalence of geographic tongue in burning mouth complaints: a retrospective study. Oral Surg Oral Med Oral Pathol Oral Radiol. 2012;114(4):444-8. doi: 10.1016/j.0ooo.2012.04.006. [PubMed: 22901641].

26. Mirzaeian R, Sadoughi F, Tahmasebian S, Mojahedi M. Progresses and challenges in the traditional medicine information system: A systematic review. J Pharm Pharmacogn Res. 2019:38-40.

27. Shams Ardekani M. Reviewing the history and foundations of Islamic and Iranian traditional medicine. Chogan. 2008:38-40.

28. Nimrouzi M, Daneshfard B, Parvizi MM. Dream in Persian medicine perspective: a narrative review. Int $j$ dream res. 2020:119-22.

29. Navidi AA, Ghasemi Soleymaniesh MA. Encyclopedia of holistic medicine traditional and modern methods in medicine. Tehran: Cultural Institute Tymvrzadh Publishers; 2003. 
30. Kord Afshari GH, Mohhamdi Kenri H, Esmaeili S. . NaslNikan; 2008. $216 \mathrm{p}$.

31. Vahedi A, Zamani M, Mojahedi M, Mozaffarpur S, Saghebi R, Mououdi M. Role of anthropometric dimensions of human body in identifying temperament in traditional Persian medicine.JBabol Univ Med Sci. 2016;18(7):24-33.

32. Nimrouzi M, Zare M. Principles of nutrition in Islamic and traditional Persian medicine. J Evid Based Complementary Altern Med. 2014;19(4):267-70. doi: 10.1177/2156587214542006. [PubMed: 25027179].

33. Nimrouzi M, Salehi A, Ahmadi A, Kiani H. Avicenna's medical didactic poem: Urjuzehtebbi.AMHA-Acta medico-historica Adriatica. 2015;13:4556.

34. Mojahedi M, Naseri M, Majdzadeh R, Keshavarz M, Ebadini M, Nazem E, et al. Reliability and validity assessment of mizaj questionnaire: A novel self-report scale in iranian traditional medicine. Iran Red Crescent Med J. 2014;16(3):15924. doi: 10.5812/ircmj.15924. [PubMed: 24829785]. [PubMed Central: PMC4005447].

35. Khozeimeh F, Taheri MA. [Evaluation of relative frequency of geographic tongue and fissured tongue in psoriatic patients]. Dent Res J(Isfahan). 2009:88-93. Persian.

36. Sarifakioglu E, Gunduz C, Gorpelioglu C. Oral mucosa manifestations in 100 pregnant versus non-pregnant patients: an epidemiological observational study. EurJ Dermatol. 2006;16(6):674-6.

37. Kullaa-Mikkonen A, Mikkonen M, Kotilainen R. Prevalence of different morphologic forms of the human tongue in young Finns. Oral Surg Oral Med Oral Pathol Oral Radiol. 1982;53(2):152-6. doi: 10.1016/00304220(82)90281-x.

38. Maleki Z, Saneei A, Darvishi Tafvizi M, Naseri F. An epidemiological study of fissured tongue in the population of Tehran Province in 1995. J Dent Sch. 2003;21(4):578-83.

39. Taheri J, Maleki Z. An epidemiological study of 28-18-year-old scrapspeaking language students in dental school students Shahid Beheshti University of Medical Sciences in 1999. J Islamic Dent Assoc Iran. 1999;4(12):85-90.
40. Neville BW, Damm DD, Allen CM. Bouquot Je. Oral and maxillofacial pathology.2nd ed. Philadelphia: WB Saunders; 2002. p. 677-8.

41. Díaz-Guzmán LM, Castellanos-Suárez JL. Lesions of the oral mucosa and periodontal disease behavior in pregnant patients. Med Oral Patol Oral Cir Bucal. 2004;9(5):430-3.

42. Alizadeh M, Khadem E, Aliasl J. Diagnosis protocol of stomach distemperament for clinical practice in Iranian traditional medicine: A narrative review. Iran J Public Health. 2017;46(7):877.

43. Kovac-Kovacic M, Skaleric U. The prevalence of oral mucosal lesions in a population in Ljubljana, Slovenia. J Oral Pathol Med. 2000;29(7):3315. doi: 10.1034/j.1600-0714.2000.290707.x. [PubMed: 10947249].

44. Parvizi MM, Nimrouzi M, Bagheri Lankarani K, Emami Alorizi SM, Hajimonfarednejad $M$. Health recommendations for the elderly in the viewpoint of traditional persian medicine. Shiraz E Med J. 2018;19(1). doi: 10.5812/semj.14201.

45. Aghili Khorasani Shirazi MH. Kholasat al-hekmah (the principal's of traditional iranian medicine). Qom, Iran: Esmaeilian; 2006.

46. Atarzadeh F, Nimrouzi M, Amin G. Blistering disease in view point of Avicenna. J Integr Med. 2016;14(6):412-4. doi: 10.1016/s20954964(16)60277-7.

47. Atarzadeh F, Kamalinejad M, Dastgheib L, Amin G, Jaladat AM, Nimrouzi M. Cassia fistula: A remedy from Traditional Persian Medicine for treatment of cutaneous lesions of pemphigus vulgaris. Avicenna J Phytomed. 2017;7(2):107.

48. Nimrouzi M, Zarshenas MM. Management of anorexia in elderly as remarked by medieval Persian physicians. Acta Med Hist Adriat. 2015;13(2):115-28.

49. Mosavat SH, Marzban M, Bahrami M, Parvizi MM, Hajimonfarednejad M. Sexual headache from view point of Avicenna and traditional Persian medicine. Neurol Sci. 2017;38(1):193-6. doi: 10.1007/s10072-0162741-4. [PubMed: 27770272].

50. Parvizi MM, Nimrouzi M, Pasalar M, Salehi A, Hajimonfarednejad M, Amini F, et al. Association between personality types and temperament (mizaj) based on persian medicine. Shiraz E Med J. 2018;19(12). doi: 10.5812/semj.68950. 